\title{
DICHROMATIC COLOR PERCEPTION IN A TWO STAGE MODEL: TESTING FOR CONE REPLACEMENT AND CONE LOSS MODELS
}

\author{
Carlos Eduardo Rodríguez-Pardo ${ }^{1}$, Gaurav Sharma ${ }^{1,2}$ \\ ${ }^{1}$ Department of Electrical and Computer Engineering \\ ${ }^{2}$ Department of Biostatistics and Computational Biology \\ University of Rochester, Rochester, NY 14627
}

\begin{abstract}
We formulate a two stage model of dichromatic color perception that consists of a first sensor layer with gain control followed by an opponent encoding transformation. We propose a method for estimating the unknown parameters in the model by utilizing pre-existing data from psychophysical experiments on unilateral dichromats. The model is validated using this existing data and by using predictions on known test images for detecting dichromacy. Using the model and analysis we evaluate the feasibility of cone loss and cone replacement hypotheses that have previously been proposed for modeling dichromatic color vision. Results indicate that the two stage model offers good agreement with test data. The cone loss and cone replacement models are shown to have fundamental limitations in matching psychophysical observations.
\end{abstract}

Index Terms - Dichromacy, color vision models, stage models, unilateral dichromacy, cone loss hypothesis, cone replacement hypothesis.

\section{INTRODUCTION}

Normal color perception is trichromatic, where the color sensations are determined by the responses of three types of photoreceptors in the eye, referred to as the $\mathrm{L}, \mathrm{M}$, and $\mathrm{S}$ cones, which are sensitive to the long, medium, and short wavelength regions of the visual spectrum, respectively. A significant fraction of the population, however, suffers from a form of color vision deficiency, referred to as dichromacy, where the functionality of one of the three cone types is missing. Based on whether the function of the $\mathrm{L}, \mathrm{M}$, or $\mathrm{S}$ cones is compromised, dichromats are classified as protanopes deuteranopes, and tritanopes, respectively. Understanding the color perception of dichromats is of interest, not only for the purpose of designing techniques for better presentation of visual information to dichromats $[1,2]$, but also for obtaining a better understating of how the human visual system works.

C.E. Rodriguez-Pardo is supported in part by a grant from Sharp Labs America and by a matching grant from New York State Office of Science, Technology and Academic Research (NYSTAR) through the Center for Emerging and Innovative Sciences (CEIS).
Knowing which type of cone is disabled, allows us to readily predict colors that are confused by each type of dichromat, i.e. we can determine which colors, appearing different to normal trichromats, appear identical to a given type of dichromat. However, these predictions of confusion provide only an incomplete characterization of dichromatic color perception. In particular, based on the aforementioned characterization alone, it is unclear how one may simulate dichromatic perception for a trichromatic observer; indeed the very question whether such a simulation is feasible at all is left unanswered.

Clues about the relation between the color perceptions of dichromats and trichromats can be obtained from unilateral dichromats, who are individuals that experience dichromacy in a single eye and have normal trichromatic color vision in the other eye. These individuals can compare the color perceptions of their two eyes and the results of such comparisons allow us to better understand how a dichromat sees the world, and also provide important information for modeling dichromatic perception. Using results from matching experiments performed by unilateral dichromats, researchers have previously developed methods for simulating dichromatic color perception for color normal observers [3-5] and explored the viability of alternative physiological hypotheses for dichromat color perception [6]. In particular, two possible cases are usually considered, depending on whether the entire population of the compromised cones is absent on the retina and therefore they should not be considered, known as the cone loss hypothesis, or the pigment in those cones is replaced with one of the remaining cone types, referred to as the cone replacement hypothesis [7].

In this paper, we present a different methodology for obtaining a model for dichromatic color vision. We begin with the commonly accepted two stage architecture for trichromatic color perception, consisting of a first sensor stage with per-channel gain control and a second opponent encoding stage $[8,9]$. We adapt this model to dichromats by dropping the non-functional cone from the first stage and replacing the second stage with a transform of suitable dimensionality whose parameters are unknown a priori. We propose a 


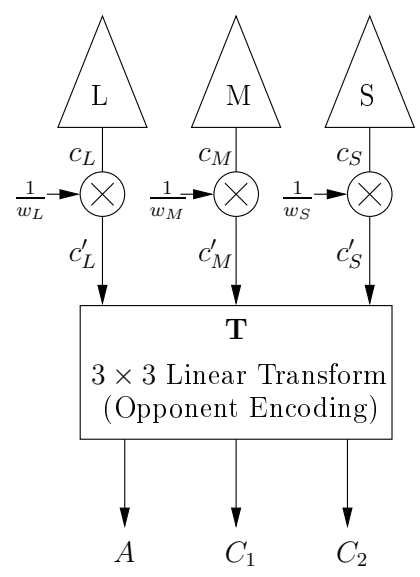

Fig. 1. Two stage model for trichromatic color vision, composed by the sensor layer (L,M,S cones) followed by gain control and opponent encoding transform.

method for estimation of these unknown model parameters using pre-existing data from matching experiments performed by unilateral dichromats. The resulting model, which allows us to relate dichromatic perception to trichromatic perception and to simulate dichromatic perception for a color normal trichromat, is validated with existing data and on images commonly employed for detecting dichromacy.

Finally, we exploit our framework to evaluate the feasibility of other hypotheses that have been previously postulated for modeling dichromatic color perception. In particular, we consider the cone loss and cone replacement hypotheses, which form models of dichromacy as reduced models of color normal vision, and evaluate their ability to predict features of dichromatic perception obtained from psychophysical experiments.

The rest of the paper is developed as follows. In Section 2 we introduce the stage model system for normal and dichromatic color vision. We present in Section 3 the method for estimating the unknown parameters in the model, and we apply it to define the second stage of our model. In Section 4 we compare our model with the loss and replacement cone hypotheses, and evaluate their feasibility. We offer a discussion of our results in Section 5, and finally we conclude in Section 6.

\section{STAGE MODELS FOR COLOR VISION}

Accepted models for color vision are usually constructed in stages, based on the physiology of human vision [8-12]. Figure 1 shows an schematic diagram that represents a two stage model.

The first stage models the functionality of the L, M, S cone, which not only sense the light, but change their sensitivity, adapting their response independently to different viewing conditions, a mechanism known as chromatic adaptation.
Following closely the notation in [13], we denote by $f(\lambda)$, the power distribution of a stimuli incident on the eye, and $s_{i}(\lambda)$ the sensitivity of the $i=L, M, S$ cones, as a functions of wavelength $\lambda$. Then, the responses of the three cones are,

$$
c_{i}=\int s_{i}(\lambda) f(\lambda) d \lambda, \quad i=L, M, S,
$$

which are collectively denoted as a tristimulus vector $\mathbf{c}=$ $\left[c_{L}, c_{M}, c_{S}\right]^{T}$. The entries of the adapted cone response vector, $\mathbf{c}^{\prime}=\left[c_{L}^{\prime}, c_{M}^{\prime}, c_{S}^{\prime}\right]^{T}$, can be found as $c_{i}^{\prime}=c_{i} / w_{i}$, where the vector $\left[w_{L}, w_{M}, w_{S}\right]^{T}$ is known as the white point, the cone response to the most bright stimuli exposed to the eye.

The second stage is represented by a $3 \times 3$ matrix $\mathbf{T}$, that transforms the data into an achromatic (light/dark) channel, $A$, and two chroma channels, $C_{1}$ and $C_{2}$ that represent opponent encodings of red-green and yellow-blue sensations. Depending on the model, different transformations, are used to represent the opponent encoding stage. These transforms share significant similarities and no single one is universally accepted. In this paper, we consider the opponent stage proposed for the spatial extension of CIELAB (S-CIELAB) [14], which is based on psychophysical experiments in [15]. The channels $A, C_{1}$ and $C_{2}$ are computed as, $\left[\begin{array}{lll}A & C_{1} & C_{2}\end{array}\right]^{T}=\mathbf{T} \mathbf{c}^{\prime}$, where

$$
\mathbf{T}=\left[\begin{array}{rrr}
0.999 & -0.106 & -0.094 \\
-0.669 & 0.742 & -0.027 \\
-0.212 & -0.354 & 0.911
\end{array}\right] \text {. }
$$

\subsection{Stage Models for Dichromatic Vision}

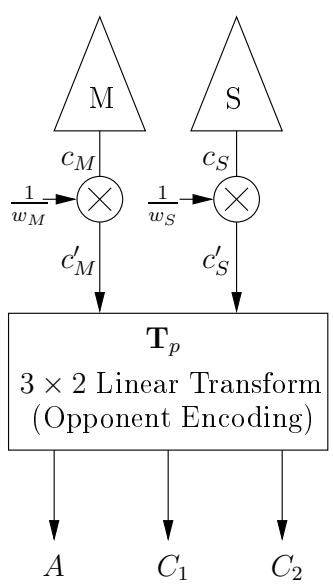

Fig. 2. Two stage model for protanopic color vision. Models for other dichromats are obtained in an analogous manner.

A architecture for a two stage model for dichromats can be intuitively deduced as a reduced version of the the model presented in Section 2. In particular, the dichromatic opponent encoding is represented by a $3 \times 2$ matrix $\mathbf{T}_{D}$, where we use $D=p, d, t$, depending on whether the protanope, deuteranope or tritanope case is considered, respectively. Figure 2 
shows an schematic for the dichromatic two stage model for the specific case of a protanope, where the L cone functionality is absent. Note that the gain control stage parameters are known from the white point, but the opponent encoding transform is unknown in this model.

\section{DICHROMATIC MODEL PARAMETER ESTIMATION METHODOLOGY}

We utilize results from matching experiments in unilateral dichromats to estimate the unknown parameters in our model. Results reported from experiments show that for each type of dichromacy it is possible to find two wavelengths regions of the visual spectrum, where the individuals register a match in color perception between both eyes for (close to) monochromatic stimuli [16-18]. Wavelengths with such characteristics are called isochromes [16]. Isochromes for protanopes and deuteranopes can be found in stimuli with wavelengths around $475 \mathrm{~nm}$ and $575 \mathrm{~nm}$, where the perceived colors are reported as blue and yellow, while for tritanopes, the isochromes are found in $485 \mathrm{~nm}$, perceived as blue-green and 660nm, which is labeled as red [3]. For unilateral dichromats, in addition to the isochromes, neutral stimuli, i.e. stimuli whose chromaticity matches that of the white point, also appear match between the normal and dichromatic eyes. We postulate that for the matches observed by unilateral dichromats, the opponent encoded representations match and use the resulting constraints to estimate our model parameters. This process is outlined next.

For each of the three types of dichromacy, we wish to estimate the $3 \times 2$ matrix $\mathbf{T}_{D}$. Denote by $\mathbf{M}_{D}$ the $3 \times 2$ matrix whose columns contain, for the dichromatic eye, the adapted cone responses for the white and the two isochromes, and let $\mathbf{M}$ be the $3 \times 2$ matrix with the adapted cone responses, for the trichromat, for the same stimuli, in the same order. Then the results of the experiments imply that if the model applies, we must have,

$$
\mathbf{T}_{D} \mathbf{M}_{D}=\mathbf{T M}
$$

We therefore estimate $\mathbf{T}_{D}$ as the matrix that offers the best approximation in (3) in a least-squares sense. Specifically, we obtain $\mathbf{T}_{D}=\mathbf{T M}\left(\mathbf{M}_{D}\right)^{+}$, where $\left(\mathbf{M}_{D}\right)^{+}$denotes the Moore-Penrose pseudo inverse matrix of $\mathbf{M}_{D}$ [19]. Using normalized the Hunt-Pointer-Estevez cone sensitivities [20], and a white-point obtained from the equi-energy stimuli ${ }^{1}$, for the protanope we obtain,

$$
\mathbf{T}_{p}=\left[\begin{array}{rr}
0.9769 & -0.1870 \\
0.0102 & 0.0358 \\
-0.5859 & 0.9309
\end{array}\right]
$$

Estimates of $\mathbf{T}_{d}$ for deuteranopes and $\mathbf{T}_{t}$ for tritanopes are obtained similarly.

\footnotetext{
${ }^{1}$ In practice, the experimental white-point deviates slightly from the equienergy stimuli. Since the exact values are not reported in all cases, we ignore these deviations pending further investigations.
}

To test the performance of the model, we first evaluate the relative error in the predicted matches for the unilateral dichromats, defined as,

$$
\epsilon_{D}=\frac{\left\|\mathbf{T}_{D} \mathbf{M}_{D}-\mathbf{T M}\right\|_{F}}{\|\mathbf{T M}\|_{F}},
$$

where $\|\cdot\|_{F}$ denotes the Frobenius norm [19]. For the the three types of dichromats, we obtain the relative errors $\left[\epsilon_{p}, \epsilon_{d}, \epsilon_{t}\right]^{T}=[0.0013,0.0008,0.0085]^{T}$. It is clear that the model provides a good fit.

As a second validation step, we use the estimated model to simulate dichromatic perception for a normal trichromatic observer and test the plausibility of the simulation using electronic versions of the Ishihara plates used for detecting dichromacy. Based on our postulate of matching in the opponent representation, we assume that for any stimulus, the model-predicted vector $\left[A, C_{1}, C_{2}\right]^{T}$ for the dichromat defines the matching trichromatic opponent representation, from which the corresponding colorimetry can be obtained by inverting the model of Fig. 1. This colorimetry presented to a normal trichromatic observer, would simulate the dichromat's color perception of the stimulus. Figure 3 shows two original Ishihara-style images ${ }^{2}$ and simulated dichromatic appearance of these images for each of the three types of dichromats. The pattern in the first images is intended to be invisible to protanopes and deuteranopes whereas the pattern in the second image is intended to be invisible to a tritanope. In all three cases, the simulated images are in good agreement with the expected results (as judged by the authors and a small a small number of additional color normal observers).

\section{TESTING FOR CONE LOSS AND CONE REPLACEMENT HYPOTHESES}

We compare our model for dichromatic color vision with other previously proposed hypotheses, by considering their ability to explain features of dichromatic perception obtained from psychophysical experiments, in particular, the matchings in color perception between trichromats and color normal observers for the white and the isochrome stimuli.

We consider the cone loss and cone replacement a hypotheses that have been used to define dichromatic models as reduced versions of trichromatic models [4, 6, 23]. Figures 5 (a) and (b) show the models that result for dichromatic color perception when the loss and replacement hypotheses, respectively, are applied within the two stage model of Fig. 1.

Clearly, the models in Figs. 5 (a) and (b), corresponding to the loss and replacement hypotheses are specific instances of our more general two-stage dichromatic model of Section 2.1, where, the loss and replacement opponent encoding transforms $\mathbf{T}_{D}^{l}, \mathbf{T}_{D}^{r}$, are given by, $\mathbf{T}_{D}^{l}=\mathbf{T} \Gamma_{D}^{l}$, and $\mathbf{T}_{D}^{r}=\mathbf{T} \Gamma_{D}^{r}$, respectively, where the matrices $\Gamma_{D}^{l}$ and $\Gamma_{D}^{r}$ represent the cone entries to the opponent coding stage, and

\footnotetext{
${ }^{2}$ The original images are obtained from [21] and [22].
} 

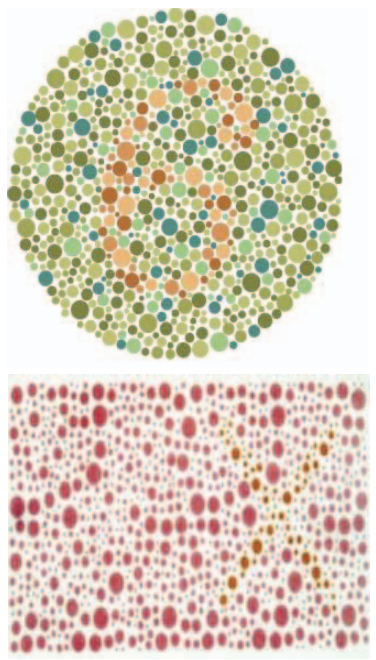

Original
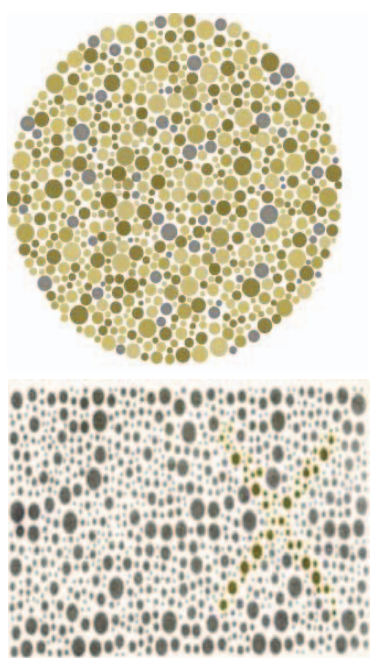

Protanope
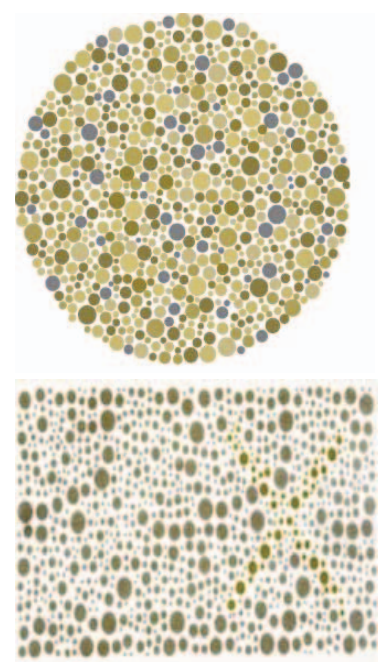

Deuteranope
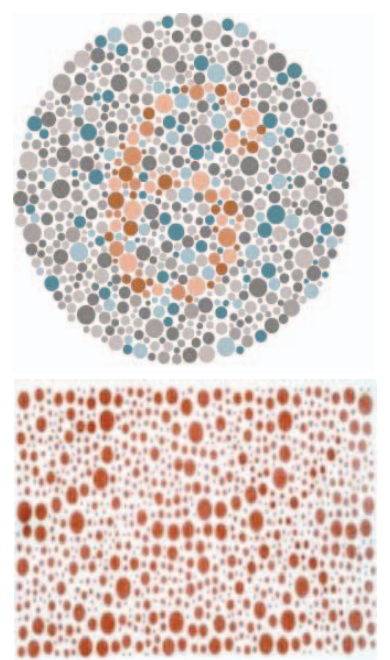

Tritanope

Fig. 3. Simulation of dichromat perception based on the proposed two stage model. The four columns from left to right present the original image and simulations of the original image for protanopes, deuteranopes, and tritanopes. Top: a Ishihara image containing the a patterned digit " 6 ", expected to be invisible to protanopes and deuteranopes. Bottom: an analogous image used for evaluation of tritanopia.

are defined as,

$$
\Gamma_{p}^{l}=\left[\begin{array}{ll}
0 & 0 \\
1 & 0 \\
0 & 1
\end{array}\right], \Gamma_{p}^{r}=\left[\begin{array}{ll}
1 & 0 \\
1 & 0 \\
0 & 1
\end{array}\right],
$$

for the protanope, and similarly for the deuteranope and tritanope.

Next we test whether the parameter estimates $\mathbf{T}_{p}, \mathbf{T}_{d}$, and $\mathbf{T}_{t}$ for the opponent encoding matrices for the dichromatic observers obtained via the method of Section 3, support the loss or replacement hypotheses. Based on the preceding observation, for each of the three dichromat types, we express the dichromatic opponent transform $\mathbf{T}_{D}$, in terms of the trichromat transformation $\mathbf{T}$. Specifically, we find a $3 \times 2$ matrix $\Gamma_{D}$ such that, $\mathbf{T}_{D}=\mathbf{T} \Gamma_{D}$, i.e. $\Gamma_{D}=\mathbf{T}^{-1} \mathbf{T}_{D}$, to obtain for the three cases

$$
\begin{aligned}
\Gamma_{p} & =\left[\begin{array}{rr}
1.0939 & -0.0939 \\
1.0000 & 0.0000 \\
0.0000 & 1.0000
\end{array}\right], \\
\Gamma_{d} & =\left[\begin{array}{ll}
1.0000 & 0.0000 \\
0.9140 & 0.0860 \\
0.0000 & 1.0000
\end{array}\right], \\
\Gamma_{t} & =\left[\begin{array}{rr}
1.0000 & 0.0000 \\
0.0000 & 1.0000 \\
-2.5250 & 3.5250
\end{array}\right] .
\end{aligned}
$$

At first glance $\Gamma_{p}$ and $\Gamma_{d}$ appear very similar to the matrices for the replacement hypothesis (see (6), for instance), while
$\Gamma_{t}$ is clearly distinct from either the loss or replacement hypotheses, although taking the difference of the $\mathrm{L}$ response from the $\mathrm{M}$ response can be appreciated as a reasonable way to obtain a correlate for the missing $\mathrm{S}$ response. Additional analysis, which we describe in the sequel, however, illustrates that the replacement hypothesis is inadequate in explaining the psychophysical observations.

To consider the ability of the replacement model to explain the observed data, we consider the Euclidean distance $\Delta E_{A, C 1, C 2}$ in the $A, C_{1}, C_{2}$ space $^{3}$, between the predicted color perceptions of monochromatic stimuli for a trichromat and a protanope ${ }^{4}$, where for the protanope the loss hypothesis induced model of Fig. 5 (a) is utilized. Figure 4 includes a plot of this Euclidean distance as a function of the wavelength of the monochromatic stimulus, which is identified in the figure legend (red dash-dot plot). The plot indicates that under the replacement hypothesis, only a single isochrome is predicted (at the wavelength where the difference $\Delta E_{A, C 1, C 2}=0$ for the replacement hypothesis plot in Fig. 4) instead of the two isochromes observed in the psychophysical experiments. The process outlined above for the protanope color perception model under the replacement hypothesis was repeated for two additional models: 1) our proposed model for the protanope with the parameter matrix $\mathbf{T}_{p}$ given in (4) and 2) for the loss hypothesis induced model of Fig. 5 (b). The plots of the color difference $\Delta E_{A, C 1, C 2}$ as

\footnotetext{
${ }^{3}$ Because we use the distance in $A, C_{1}, C_{2}$ space to infer only matches or mismatches, the lack of perceptual uniformity is not a serious limitation.

${ }^{4}$ Results for deuteranope and tritanope are similar.
} 


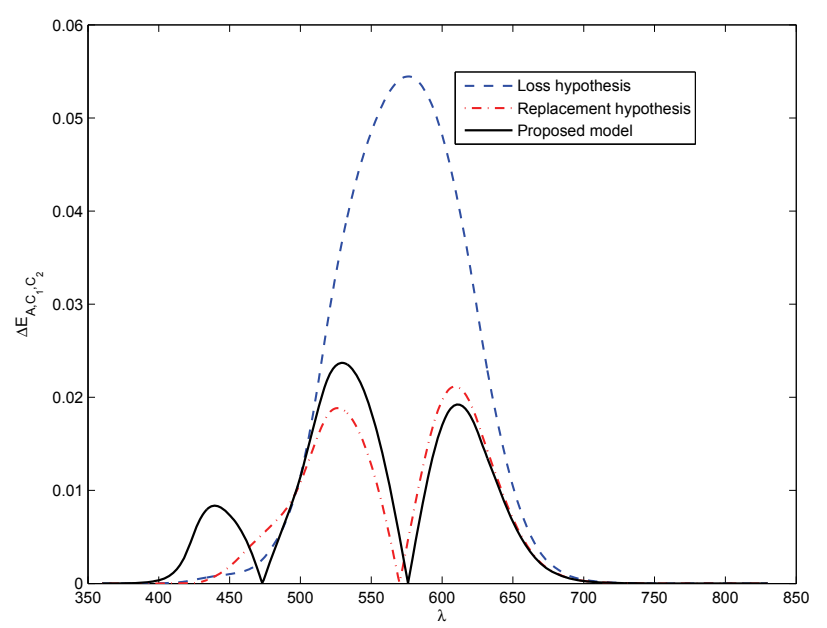

Fig. 4. Difference between the color perception of a trichromat and a protanope, quantified as the Euclidean distance $\Delta E_{A, C 1, C 2}$ in the $A, C_{1}, C_{2}$ space for monochromatic stimuli, assuming: the loss model (dashed line), the replacement model (dashed-dot line) the proposed model with the estimated opponent transform $\mathbf{T}_{p}$ (solid line).

a function of the wavelength of the monochromatic stimulus for these two cases are also included in Fig. 4 and identified in the figure legend. In these plots, we see that the proposed model clearly predicts two isochrome matches at the correct wavelengths whereas the loss model predicts no isochromes. In fact, with a little algebra, one can analytically establish that under the loss hypothesis, perceptual matchings between a color normal trichromat and dichromat happen only when the stimulus of light that produces, in the trichromat, a cone response $c_{i}^{\prime}=c_{i}=0$, depending on which $i=L, M, S$ cone is missing. This implies that only trivial isochromes, i.e. wavelengths for which the compromised cone produce no response, are predicted by this model.

The clear differences of the models in their ability to explain the psychophysical data in all the forms of dichromacy, suggest that the differences, in particular the small nonzero entries in $\Gamma_{p}$ and $\Gamma_{d}$ in (7), play an important role and the pure loss or replacement hypotheses appear inadequate.

\section{DISCUSSION}

The methodology we have utilized for obtaining our two stage model, relies on data from matches perceived by unilateral dichromats and relies on the assumption that unilateral perception does represent the perception in the dichromat population. This assumption is hard to justify rigorously due to the difficulty of comparing the perceptions of different individuals. For the same reasons, however, the assumption represents one of the few workable methods for modeling dichromatic

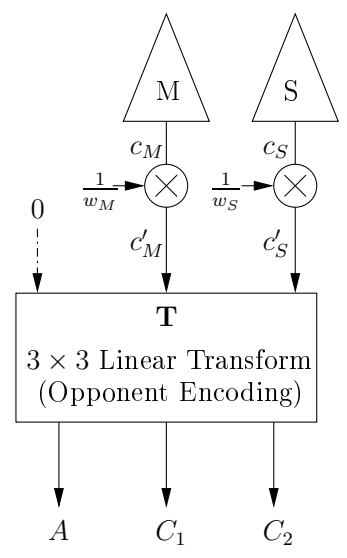

(a)

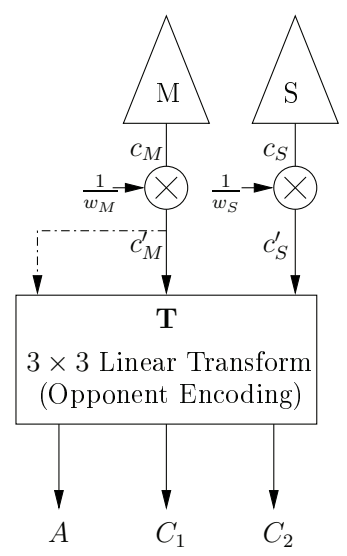

(b)
Fig. 5. Protanopic color perception models resulting from (a) Cone loss and (b) Cone replacement, in the model of Fig. 1.

color perception and is also extensively utilized in other work on modeling dichromatic color perception. It should be recognized that the assumption neglects neural adaptations that unilateral dichromats may develop in order to overcome the dissimilarity between the information provided by the two eyes. Also dichromacy may be encountered as the result of different underlying causes, which may manifest themselves in different ways and our analysis and modeling may therefore not be universal.

We made the, also reasonable, assumption that the both eyes have cone sensitivities with the same characteristics, with the clear exception of the compromised cone, and even more, we used a standard observer. Using genetics analysis, it is possible nowadays to determine precisely for an individual the family of cone responses the observer exhibits, and the color visual deficiency it may posses [24]. Moreover, it is possible to estimate the cone mosaic without invasive methods and determine physiologically the predominance of one cone model hypothesis over the other [25]. Such tools were not available by the time the psychophysical experiments on unilateral individuals considered here were performed, and certainly that information would enrich the model. On the contrary the information we have about the unilateral dichromats may not be completely accurate. The classification of individuals for that experiments were based on classical procedures like pseudo-isochromatic plates or Farnsworth-Munsell test [26], that although highly precise, do not exclude the possibility errors in the classification of the individuals, specially between dichromats and anomalous trichromats.

Despite these limitations, the model we introduce in this paper has various advantages. In addition to the benefits we have already outlined, the proposed model also offers the philosophical advantage that it does not $a$ priori assume either 
the cone loss or cone replacement hypothesis. Until recently, these two hypotheses were considered mutually exclusive options but are now believed to be both physiologically feasible in the human eye. Estimations of the cone mosaic in [27,28], support the replacement, while [25] shows evidence that functional photoreceptor loss is possible in individuals of certain genotypes. In addition, the replacement hypothesis is normally discarded for tritanopia because of the significant differences between the genes that define the pigments of the $\mathrm{S}$ cone and the genes for the $\mathrm{L}$ and $\mathrm{M}$ cones [7].

\section{CONCLUSIONS AND FUTURE WORK}

In this paper, we proposed a methodology for modeling color perception of dichromats by formulating a two-stage model and estimating the model parameters using known match conditions for unilateral dichromats. The models obtained with the proposed methodology offer agreement with the perceptual data and also offer plausible predictions for images used for testing for dichromacy. Furthermore, we also introduced a framework to evaluate dichromatic models of color perception, and showed that the models induced by the cone replacement and loss hypothesis appear to have fundamental limitations.

Promising as our results are, they would be significantly strengthened by psychophysical validation, which we have not attempted in this initial phase of our work because of the lack of a suitable and readily accessible pool of dichromatic observers. We plan to address this validation in a continuation of our work.

\section{REFERENCES}

[1] C. E. Rodríguez-Pardo and G. Sharma, "Adaptive color visualization for dichromats using a customized hierarchical palette," in Proc. SPIE: Color Imaging XVI: Displaying, Processing, Hardcopy, and Applications, R. Eschbach, G. G. Marcu, and A. Rizzi, Eds., vol. 7866, Jan. 2011, pp. 7866-2,1-9.

[2] J. Huang, Y. Tseng, S. Wu, and S. Wang, "Information preserving color transformation for protanopia and deuteranopia," IEEE Sig. Proc. Lett., vol. 14, no. 10, pp. 711-714, 2007.

[3] H. Brettel, F. Viénot, and J. Mollon, "Computerized simulation of color appearance for dichromats," J. Opt. Soc. Am. A, vol. 14, no. 10, pp. 2647-2655, 1997.

[4] G. Machado, M. Oliveira, and L. Fernandes, "A physiologically-based model for simulation of color vision deficiency," IEEE Transactions on Visualization and Computer Graphics, pp. 1291-1298, 2009.

[5] G. Meyer and D. Greenberg, "Color-defective vision and computer graphics displays," IEEE Comp. Graphics and Applic., pp. 28-40, 1988.

[6] P. Capilla, M. Luque, and M. Díez-Ajenjo, "Looking for the dichromatic version of a colour vision model," Journal of Optics A: Pure and Applied Optics, vol. 6, pp. 906-919, 2004.

[7] K. Gegenfurtner and L. Sharpe, Color vision: from genes to perception. Cambridge University Press, 2001.

[8] S. Guth, R. Massof, and T. Benzschawel, "Vector model for normal and dichromatic color vision," J. Opt. Soc. Am., vol. 70, no. 2, pp. 197-212, 1980.
[9] C. Ingling Jr and B. Tsou, "Orthogonal combination of the three visual channels," Vis. Res., vol. 17, no. 9, pp. 1075-1082, 1977.

[10] R. Massof and J. Bird, "A general zone theory of color and brightness vision. I. Basic formulation,” JOSA, vol. 68, no. 11, pp. 1465-1471, 1978.

[11] R. De Valois and K. De Valois, "A multi-stage color model," Vis. Res., vol. 33, no. 8, pp. 1053-1065, 1993.

[12] L. Guth, "Model for color vision and light adaptation," J. Opt. Soc. Am. A, vol. 8, no. 6, pp. 976-993, 1991.

[13] G. Sharma and H. J. Trussell, "Digital color imaging," IEEE Trans. Image Proc., vol. 6, no. 7, pp. 901-932, Jul. 1997. [Online]. Available: http://www.ece.rochester.edu/ /gsharma/papers/dciip97.pdf

[14] X. Zhang, B. Wandell, et al., "A spatial extension of CIELAB for digital color-image reproduction," Journal of the Society for Information Display, vol. 5, no. 1, pp. 61-64, 1997.

[15] K. Bäuml and B. Wandell, "Color appearance of mixture gratings," Vis. Res., vol. 36, no. 18, pp. 2849-2864, 1996.

[16] M. Alpern, K. Kitahara, and D. Krantz, "Perception of colour in unilateral tritanopia." The Journal of Physiology, vol. 335, no. 1, p. 683, 1983.

[17] D. Judd, "The color perceptions of deuteranopic and protanopic observers," J. Opt. Soc. Am., vol. 39, no. 3, p. 349, 1979.

[18] D. MacLeod and P. Lennie, "Red-green blindness confined to one eye," Vis. Res., vol. 16, no. 7, pp. 691-702, 1976.

[19] A. Laub, Matrix analysis for scientists and engineers. SIAM, 2005.

[20] R. W. G. Hunt and M. R. Pointer, "A color-appearance transform for the CIE 1931 standard colorimetric observer," Color Res. Appl., vol. 10, no. 3, pp. 165-179, Fall 1985.

[21] "Ishihara style image," http://offsetpressman.blogspot.com/2009/10/colorblindness-test-for-printers.html, accessed Feb. 2011.

[22] A. Landers, I. Murdoch, J. Birch, S. Cousens, O. Babalola, B. Lawal, A. Abiose, and B. Jones, "Blue-yellow colour vision in an onchocercal area of northern Nigeria," British Medical Journal, vol. 82, no. 5, p. 510, 1998. [Online]. Available: http://bjo.bmj.com/content/82/5/510.full

[23] P. Capilla, M. Díez-Ajenjo, M. Luque, and M. Jesús, "Correspondingpair procedure: a new approach to simulation of dichromatic color perception," J. Opt. Soc. Am. A, vol. 6, pp. 176-186, 2004.

[24] J. Neitz and M. Neitz, "The genetics of normal and defective color vision," Vis. Res., vol. In Press, Corrected Proof, 2011.

[25] J. Carroll, M. Neitz, H. Hofer, J. Neitz, and D. Williams, "Functional photoreceptor loss revealed with adaptive optics: an alternate cause of color blindness," Proc. Natl. Acad. Sci. USA, vol. 101, no. 22, p. 8461, 2004.

[26] D. Farnsworth, "The Farnsworth-Munsell 100-hue and dichotomous tests for color vision," J. Opt. Soc. Am., vol. 33, no. 10, pp. 568-574, 1943.

[27] J. Kremers, T. Usui, H. Scholl, and L. Sharpe, "Cone signal contributions to electrograms in dichromats and trichromats," Investigative ophthalmology \& visual science, vol. 40, no. 5, pp. 920-930, 1999.

[28] T. Berendschot, J. Van de Kraats, and D. Van Norren, "Foveal cone mosaic and visual pigment density in dichromats." Journal of Physiology, vol. 492, no. 1, pp. 307-314, 1996. 